\title{
MS39-P01 | DEVELOPMENT OF DATA PROCESSING METHOdS IN TIME-RESOLVEd LAUE
}

\section{PHOTOCRYSTALLOGRAPHY}

Kaminski, Radoslaw (Department of Chemistry, University of Warsaw, Warsaw, POL); Szarejko, Dariusz (Department of Chemistry, University of Warsaw, Warsaw, POL); Laski, Piotr (Department of Chemistry, University of Warsaw, Warsaw, POL); Jarzembska, Katarzyna (Department of Chemistry, University of Warsaw, Warsaw, POL)

Time-resolved X-ray Laue photocrystallography have recently become a promising experimental method for elucidation of short-lived species' structures in molecular crystals. Recent successful examples include determination of excited state structures in rhodium,[1] copper [2] or bimetallic [3] coordination complexes. In all of these cases the required data quality was achieved via application of very short (down to 80 ps) and intense Xray pulses, available only at synchrotron beamlines using 'pink' $X$-ray beam. However, the use of polychromatic radiation imposes some difficulties in further data processing. Some of these problems have recently been actively studied and to some extent overcome, especially in the case of data indexing.[4] Nevertheless, some aspects of data processing methods are still in their infancy and further progress is needed. Hence, here we present our most recent developments in data processing concerning the integration methods Our approach is tested on several real-life examples of data collected at APS (USA) or ESRF (France) synchrotron sources.

The authors thank NSC (2014/15/D/ST4/02856, 2016/21/D/ST4/03753) and WCSS (grant No. 285) in Poland, and APS (DOE: DE-AC02-06CH11357, NIH: R24GM111072) and ESRF, for financial support and access to facilities.

[1] J. B. Benedict et al., Chem. Commun. 2011, 47, 1704.

[2] (a) A. Makal et al. J. Phys. Chem. A 2012, 116, 3359; (b) P. Coppens et al., Phys. Scr. 2016, 91, 023003;

[3] K. N. Jarzembska, R. Kamiński et al., Inorg. Chem. 2014, 53, 10594.

[4] J. A. Kalinowski et al., J. Appl. Cryst. 2011, 44, 1182. 